\title{
A 60-month follow-up of a naturalistic study of integrative treatment for real-life geriatric patients with depression, dementia and multiple chronic illnesses*
}

\author{
Valentin Bragin ${ }^{1 \#}$, Marina Chemodanova ${ }^{1}$, Ilya Bragin ${ }^{1,2}$, Narmina Dzhafarova ${ }^{1}$, Irina Mescher ${ }^{1}$, \\ Pavlo Chernyavskyy ${ }^{1}$, Mark E. Obrenovich ${ }^{3}$, Hector H. Palacios ${ }^{4}$, Gjumrakch Aliev ${ }^{1,5,6 \dagger}$ \\ ${ }^{1}$ Stress Relief and Memory Training Center, New York, USA \\ ${ }^{2}$ Upstate Medical University, Syracuse, USA \\ ${ }^{3}$ Cleveland State University, Cleveland, USA \\ ${ }^{4}$ National Institute on Aging, Baltimore, USA \\ ${ }^{5}$ School of Health Science and Healthcare Administration, University of Atlanta, Atlanta, USA \\ “"GALLY” International Biomedical Research Institute Inc., San Antonio, USA \\ Email: ${ }^{*}$ aliev03@gmail.com, ${ }^{*}$ cobalt55@gallyinternational.com, ${ }^{\dagger}$ val11235@yahoo.com, ${ }^{\dagger}$ val11235@gmail.com
}

Received 28 January 2012; revised 29 February 2012; accepted 17 March 2012

\begin{abstract}
Background: In the past we have shown the preservation and improvement of cognitive tasks in depressed and demented patients after 24 and 36 months of combined pharmacological and non-pharmacological treatment. Here we present the results of our ongoing, naturalistic study, in the same outpatient setting, at 60 month follow up. Materials and Methods: The study group consisted of 156 medically ill, physically disabled patients with mild to moderate dementia and depression. Patients were treated with antidepressants, cholinesterase inhibitors, and NMDA antagonists, along with their regular medication regimen. Non-pharmacological intervention was centered on a home-based program of physical and cognitive exercises paired with vitamins and supplements (multivitamins, vitamin E, L-methylfolate, alphalipoic acid, acetyl-L-carnitine, omega-3, and coenzyme Q-10) and diet modification. Cognitive assessments were performed yearly. Results: After 60 months of treatment, performance of all tasks remained at or above baseline. The MMSE, Cognistat-Attention, CognistatJudgment, and RFFT-Total Unique Designs demonstrated significant improvement. Conclusion: Our results, for the first time, demonstrate arrest in cognitive decline in demented/depressed patients with multiple medical co-morbidities for 60 months. Future investigations addressing the application of a combined, integrative treatment model are warranted.
\end{abstract}

Keywords: Dementia; Depression; Alzheimer Disease; Vascular Dementia; Naturalistic Observational Study;

${ }^{*}$ Conflicts of Interest: all authors have no conflicts of interest.

${ }^{\# \dagger}$ Corresponding authors.
Integrative Treatment; Non Pharmacological

Interventions; Physical Exercises; Memory Training;

Cardiovascular Diseases

\section{INTRODUCTION}

There is an ever evolving body of evidence which is reshaping our conception of the pathogenesis of Alzheimer dementia (AD) [1-5]. In addition to the amyloid model of $\mathrm{AD}$, other models of this disease have been developed $[6,7]$.

Among many pathophysiological processes, cardiovascular abnormalities, and vasculature changes have been shown to have a strong negative impact on the development and progression of $\mathrm{AD}[3,8]$. In this regard cardiovascular pathology is accompanied by changes in regional cerebral blood flow (CBF) [9,10], hypoperfusion [11-14], chronic hypoxia [15-17], white matter lesions [18], nitrogen oxide and free radical accumulation, oxidative stress [19], and mitochondrial dysfunction [20]. Theses changes have been shown to be detrimental to energy metabolism with deleterious effects on protein synthesis, neuronal function, cognitive speed, gait, coordination, and global cognition. Based on these developments and emerging neuroplasticity data, novel integrative treatment approaches for the prevention and therapy of AD have gained significant attention in recent years [8, 21-27].

In real life clinical practice, people with dementia, particularly with $\mathrm{AD}$, generally are given medications (cholinesterase inhibitors or NMDA receptors antagonists) with little to no non-pharmacological intervention. Treatments that involve solely medication show only a transient cognitive improvement with subsequent decline 
below baseline levels [28,29].

Research into combining medications with non-pharmacological interventions for $A D$ is in the fledgling stages of its development. Recent studies suggest that the combination of medications alongside non-pharmacological interventions could be more effective in treating $\mathrm{AD}$ than medication alone. In these studies, medication treatment was accompanied by one or two non-pharmacological intervention modalities, including cognitive rehabilitation [30,31], vitamin or nutriceutical supplementation [32], and physical exercises [33,34].

There have been no comprehensive studies hitherto which have investigated the long-term ( $>2$ years) effect of an integrative therapy model with pharmacological and non-pharmacological treatment modalities implemented simultaneously in a real life clinical setting.

We have been testing the hypothesis that this type of integrative therapy could delay the progression of cognitive deficit in demented, depressed and medically ill seniors in real-life clinical settings. The preservation and, in some cases, improvement of cognitive functioning relative to baseline levels in demented patients by 12,24 , and 36 months of treatment was shown in our previous work $[35,36]$.

The aim of this study is to investigate the effectiveness of this integrative treatment model for arresting cognitive decline in demented, depressed patients in outpatient settings for duration of 60 months.

\section{MATERIALS AND METHODS}

\subsection{Study Design and Patient Selection}

The observational study design was consistent with actual treatment practices. An informed consent was obtained from each patient. Clinical assessments, organic work-ups, and evaluation of cognitive functions were conducted during the first two to three office visits. These patients underwent yearly cognitive retesting to assess the progress of the treatment and to make necessary adjustments. The optimal combination of medications and non-pharmacological interventions were individually tailored to every person based on their diseases, medication profile, cognitive performance, and physical abilities/limitations.

The study group consisted of 156 geriatric patients (79 male, 77 female) with an average age of $73.7 \pm 5.43$, who were afflicted with concomitant mild to moderate dementia and depression, along with multiple medical problems (hypertension, coronary artery disease, hyperlipidemia, diabetes, arthritis, and others). Their psychiatric diagnoses were probable $\mathrm{AD}$, Vascular Dementia (VaD), or mixed dementia, and Organic Mood Disorder with Depressed Mood, Organic Anxiety Disorder and Insomnia.
This ongoing, naturalistic study is based on data that has been continuously collected from the patients' medical records during the period of 2000-2010. All data from patients who initiated treatment during this time period and remained in treatment for at least one year was included. The data were analyzed for up to 60 months of treatment. Some patients missed yearly testing and were tested in subsequent years.

Our integrative treatment protocol was designed as a combination of medication and non-pharmacological modalities. The non-pharmacological intervention included mild physical activities, cognitive training, sensory stimulation, vitamins, supplements, and dietary modification.

During the course of the study period, newly released medications (escitalopram, rivastigmine patch and memantine), vitamins and supplements (vitamin D and Lmethylfolate) were added to the treatment regimen. Medications included antidepressants (sertraline, citalopram, escitalopram, or venlafaxine XR alone or in combination with bupropion XR), cholinesterase inhibitors (donepezil, rivastigmine, or galantamine alone or in combination with memantine) along with regular medications for their medical comorbidities. Patients were given medications to manage insomnia (zolpidem, zaleplon, eszopiclone), anxiety (lorazepam, oxazepam), neuropathic pain (gabapentin), and behavioral problems (risperidone, haloperidol, quetiapine, aripiprazole) when clinically appropriate.

Non-pharmacological interventions included vitamins and supplements (multivitamins, vitamin E, vitamin D, folic acid or L-methylfolate, alpha-lipoic acid, acetyl--carnitine, omega-3 and coenzyme Q-10) and the Bragin Brain Activation Program ${ }^{\mathrm{TM}}$ (B-BAP ${ }^{\mathrm{TM}}$ ).

The B-BAPTM consists of a combination of mild sensory motor exercises and cognitive training presented in a workbook format [37]. The workbook, comprised of a description of protocols for home use, was distributed to each patient. The physical part of the program was specifically designed for people with extremely limited physical capacities including but not limited to: problems with gait and ambulation, movement restrictions due to osteoarthritis, and low cardiovascular reserve. The cornerstone of the physical activities is a series of hand and finger movements with tennis balls. A dual task component consisted of hand movements coupled with counting and breathing. Practical demonstration of the exercises was introduced to the patients and their care-takers on initial visits. Patients were encouraged to do these exercises at home several times per day, mainly in 10 to 12 minute intervals. Attention and memory training were performed with simple pen and pencil exercises at home, and supplemented with cognitive training using our originally designed computer software at our center [38, 
39].

\subsection{Neuropsychological Test}

Our neuropsychological battery consisted of 7 tests:

1) The Folstein Mini Mental Status Exam (MMSE) with a maximum score of 30 . For attention assessment serial 7's calculation was used [40].

2) Clock Drawing Test (CDT).

3) Verbal Fluency Tests (VFT)-categories included animals and letters.

4) Neurobehavioral Cognitive Status Examination (Cognistat), which is used along with MMSE to assess different cognitive domains: orientation, attention (digit span), language abilities (comprehension, repetition, naming), construction abilities, word memory (four items), calculations, similarities, and judgment [41].

5) Ruff Figural Fluency Test (RFFT), which provides information regarding nonverbal capacity of the right frontal lobe to produce unique designs [42].

6) Ruff $2 \& 7$ Selective Attention Test (Ruff 2 \& 7 SAT), which is used to assess sustained and selective visual attention [43].

7) Word List Memory Learning Test (WLMLT), which consists of a learning portion of the test, followed by 5-minute recall and recognition.

Exclusion criteria included patients younger than 65, those with severe dementia (MMSE < 13), severe depression, any evidence of psychosis, a history of alcohol or substance abuse, severe neurological disorders, and recent major stress (e.g. death in the family, surgery).

\subsection{Statistical Analysis}

The Data Analysis was carried out with SPSS for Windows, version 17.0 (SPSS Inc., Chicago, Ill.) [44]. Analysis included descriptive and non-parametric statistics (the Wilcoxon signed-rank test, two related samples). All statistical testing was two-tailed with a $\mathrm{p}$ value of $<0.05$ considered statistically significant.

\section{RESULTS}

Demographics and clinical characteristics were characterized in Table 1. The study included 77 women and 79 men with mean age of 73.7 and mean education of 12.7 years. All the participants had depression and memory problems. The average duration of depression and memory impairment were over 3 years. Over $80 \%$ of the cohort suffered from anxiety and insomnia. A majority of patients suffered from hypertension, dyslipidemia, and coronary artery disease, while a significant number had diabetes mellitus, history of head trauma, prior stroke, osteoarthritis, thyroid dysfunction, cancer and COPD.
Table 1. Demographics and clinical profile of patients.

\begin{tabular}{|c|c|c|}
\hline Demographics & Patients & Percentage \\
\hline Men & 79 & 50.64 \\
\hline \multirow[t]{2}{*}{ Women } & 77 & 49.36 \\
\hline & Mean & Standard Deviation \\
\hline Age & 73.65 & 5.43 \\
\hline Education (Years) & 12.71 & 2.91 \\
\hline \multicolumn{3}{|l|}{ Duration of Illnesses (Months) } \\
\hline Depression & 39.00 & 22.87 \\
\hline Memory & 37.85 & 3.20 \\
\hline Diagnosis & Patients & Percentage of Group \\
\hline Anxiety & 130 & 83.33 \\
\hline Insomnia & 128 & 82.05 \\
\hline Hypertension & 133 & 85.26 \\
\hline High Cholesterol & 112 & 71.79 \\
\hline Coronary Artery Disease & 91 & 58.33 \\
\hline Diabetes & 56 & 35.90 \\
\hline Arthritis & 53 & 33.97 \\
\hline Stroke & 26 & 16.67 \\
\hline Head Trauma & 29 & 18.59 \\
\hline Thyroid Disorder & 18 & 11.54 \\
\hline Cancer & 18 & 11.54 \\
\hline COPD & 15 & 9.62 \\
\hline Parkinsonism & 10 & 6.41 \\
\hline Renal Insufficiency & 10 & 6.41 \\
\hline Anemia & 6 & 3.85 \\
\hline Radiology & Patients & Percentage of Group \\
\hline Normal & 17 & 23.9 \\
\hline Abnormal & 54 & 76.1 \\
\hline
\end{tabular}

Neuroimaging data (mainly, non contrast MRI) was available for nearly half the sample. A "normal" scan was found in $24 \%$ of the imaged group while the remaining $76 \%$ were found to have cortical atrophy, lacunar infarcts, ventricular dilation, and white matter microvascular changes alone or in combination with each other.

The patient flow/testing diagram is shown in Figure 1. The percentage of the patients missing yearly testing ranged from $20.5 \%$ (by 12 months of the treatment) to $42.3 \%$ (by 60 months of the observation period). Patient drop out rate ranged from $21 \%$ - $28 \%$ between each year 


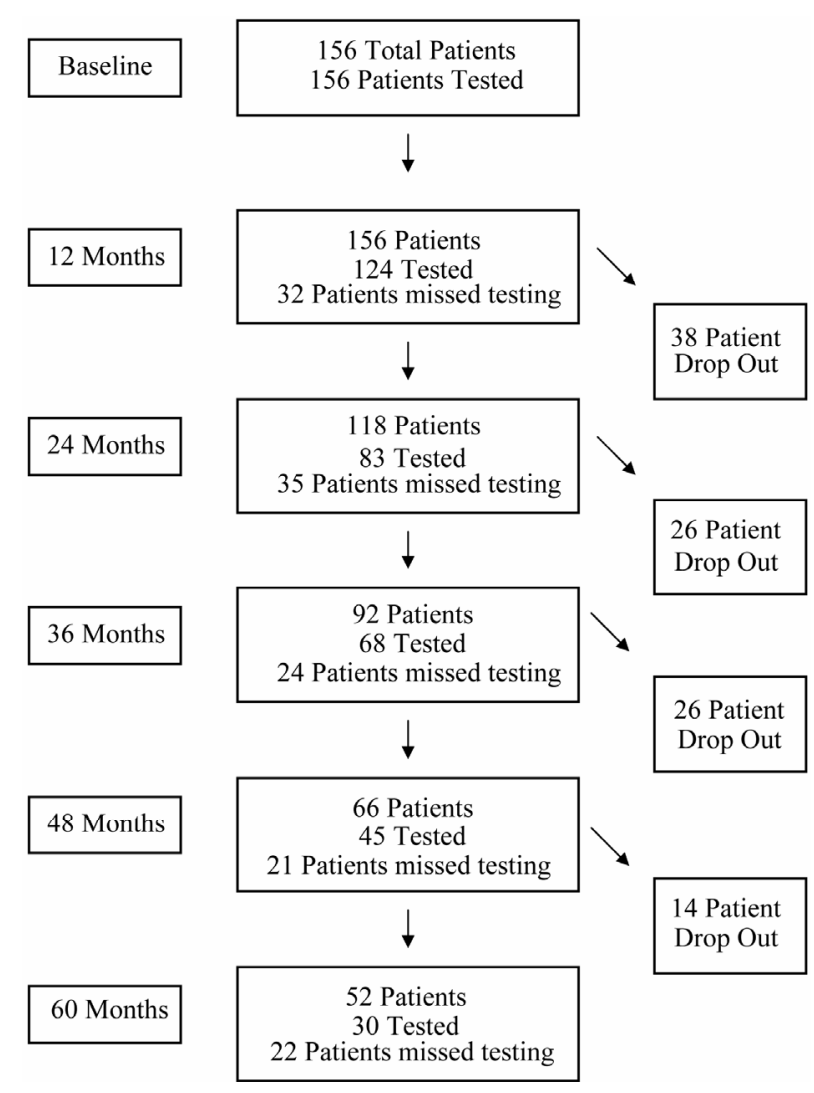

Figure 1. Patient flow/testing diagram* . *Patients tested refers to those who took the MMSE during that time testing interval. Patient drop out includes patients who left treatment for any reason including illness, death, relocation and discontinuation of therapy.

of testing.

Descriptive statistics are shown in Table 2. The MMSE score was above the baseline for the whole period of treatment with improvement ranging from $2.4 \%$ after the first year to $5.9 \%$ after 60 months (Figure 2). Performance on the CDT increased by $13.6 \%$ after 12 months, increasing to $25.0 \%$ above baseline for the next two years and decreased to $17.2 \%$ by the end of the observation period (Figure 3). The VFT (animals) score increased by a maximal amount, $14.9 \%$ above baseline at 36 months of observation and dropped back to baseline level by 60 months (Figure 4). The VFT (letters) score showed similar dynamics as the VFT (animals) (Figure $5)$.

In Cognistat, performance in all subtests for the entire period of the treatment increased. By the end of 12 months, performances in all subtests of Cognistat were significantly above the baseline, especially, in attention, construction and memory $(16.7 \%, 16.4 \%$ and $15.3 \%$ accordingly). By the end of 24 months, performances in 9 out of 10 subtests of Cognistat (except for Calculation) remained significantly above the baseline, especially, in attention, construction and memory $(26.1 \%, 24.4 \%$ and $23.9 \%$ respectively). By the end of 36 months, performances in 7 out of 10 subtests of Cognistat (except for Naming, Calculation, Similarities) remained significantly above the baseline, especially, in the same subtests: attention (28.2\%), construction (19.6\%) and memory (19.3\%). By the end of 48 months, performances in 4 out of 10 subtests of Cognistat (Orientation, Attention, Repetition and Construction) remained significantly above the baseline, especially, in attention (25.8\%) and construction (36.4\%). By the end of 60 months, performances in 2 out of 10 subtests of Cognistat (Attention and Judgment) remained significantly above the baseline, especially, in attention (27.0\%).

The Ruff 2 \& 7 SAT (Automatic Detection Speed) did not show significant decline for the whole period of observation.

The RFFT (Total Unique Designs) increased significantly at 12,36 and 60 months of the treatment $(10.1 \%$, $21.1 \%$ and $12.5 \%$ accordingly). The RFFT (Total Errors) decrease significantly only by 24 months of the treatment (17.3\%).

WLMTL did not show significant changes in performance scores, related to trial one correct, 5 minute recall and recognition.

\section{DISCUSSION}

The preservation of cognitive function in demented patients including $\mathrm{AD}$ becomes visible based on intensive basic and clinical dementia research, which expands our understanding of $\mathrm{AD}$ pathophysiology [45,46]. Among different pathophysiological factors, a reduction of brain blood flow with subsequent development of a chronic hypoxic state, oxidative stress, mitochondria failure, energy deprivation, and increased inflammation are seen [4, 12,47,48].

Prospective animal studies presented positive data related to the possible modification of the natural course of dementia. The combination of acetyl-L-carnitine and lipoic acid (ALCAR + LA) ameliorated mitochondrial dysfunction in aging rats [49] and improved learning abilities in aging beagles [50]. An antioxidant-enriched diet partially reversed mitochondrial dysfunction in aging canines [51]. In addition, the combination of an antioxidant-fortified diet and behavioral enrichment (physical exercises, social interaction, and cognitive training) improved cognitive performance in aging dogs and reduced beta amyloid (A $\beta$ ) deposition [52]. An adaptation to hypobaric intermittent hypoxia in rats with experimental AD prevented Nitrogen Oxide (NO) overproduction and ameliorated oxidative stress [53]. Moreover, recently we've demonstrated, for the first time, the agedependent effects of human Apolipoprotein E4 (ApoE4) 


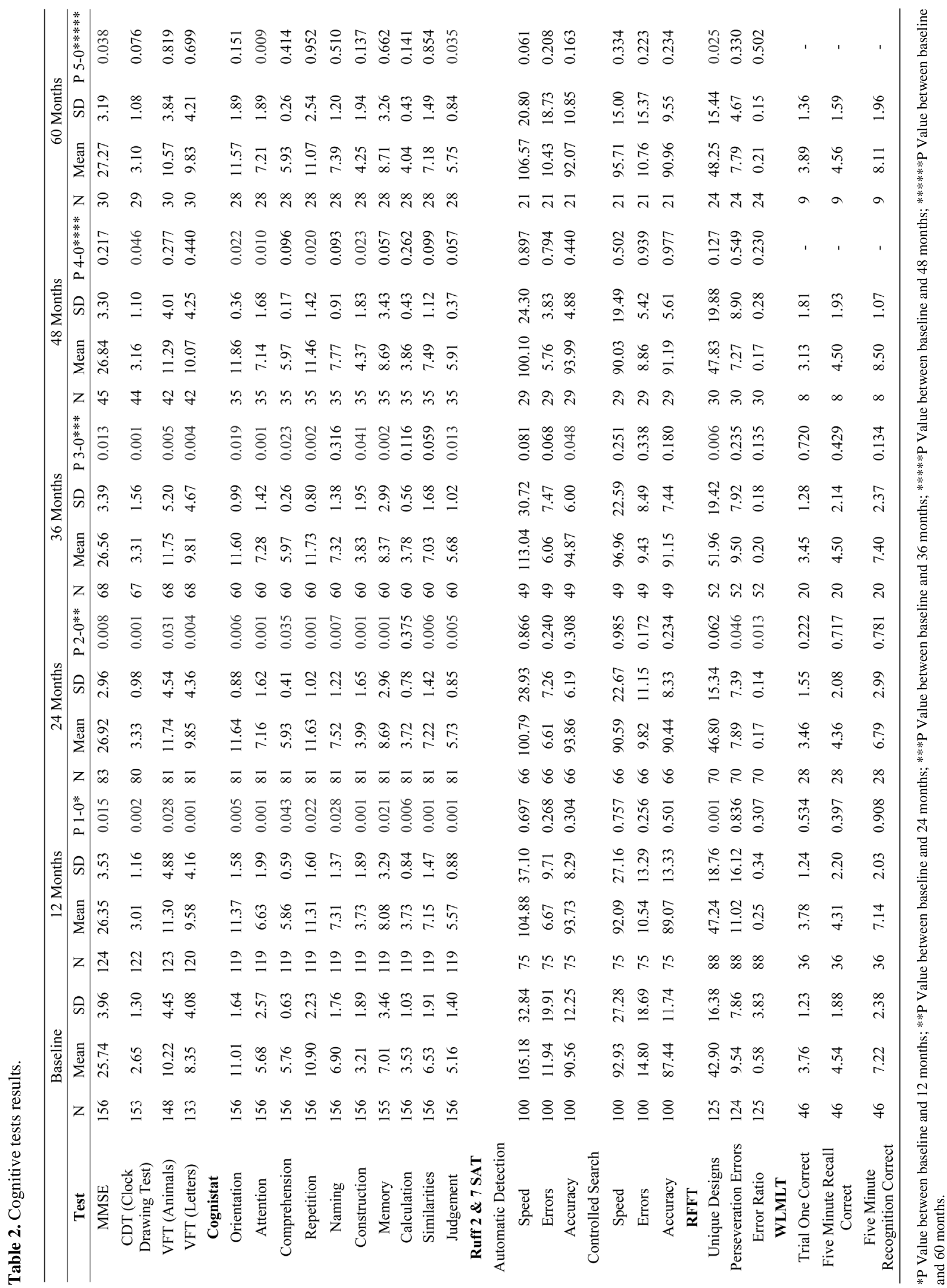




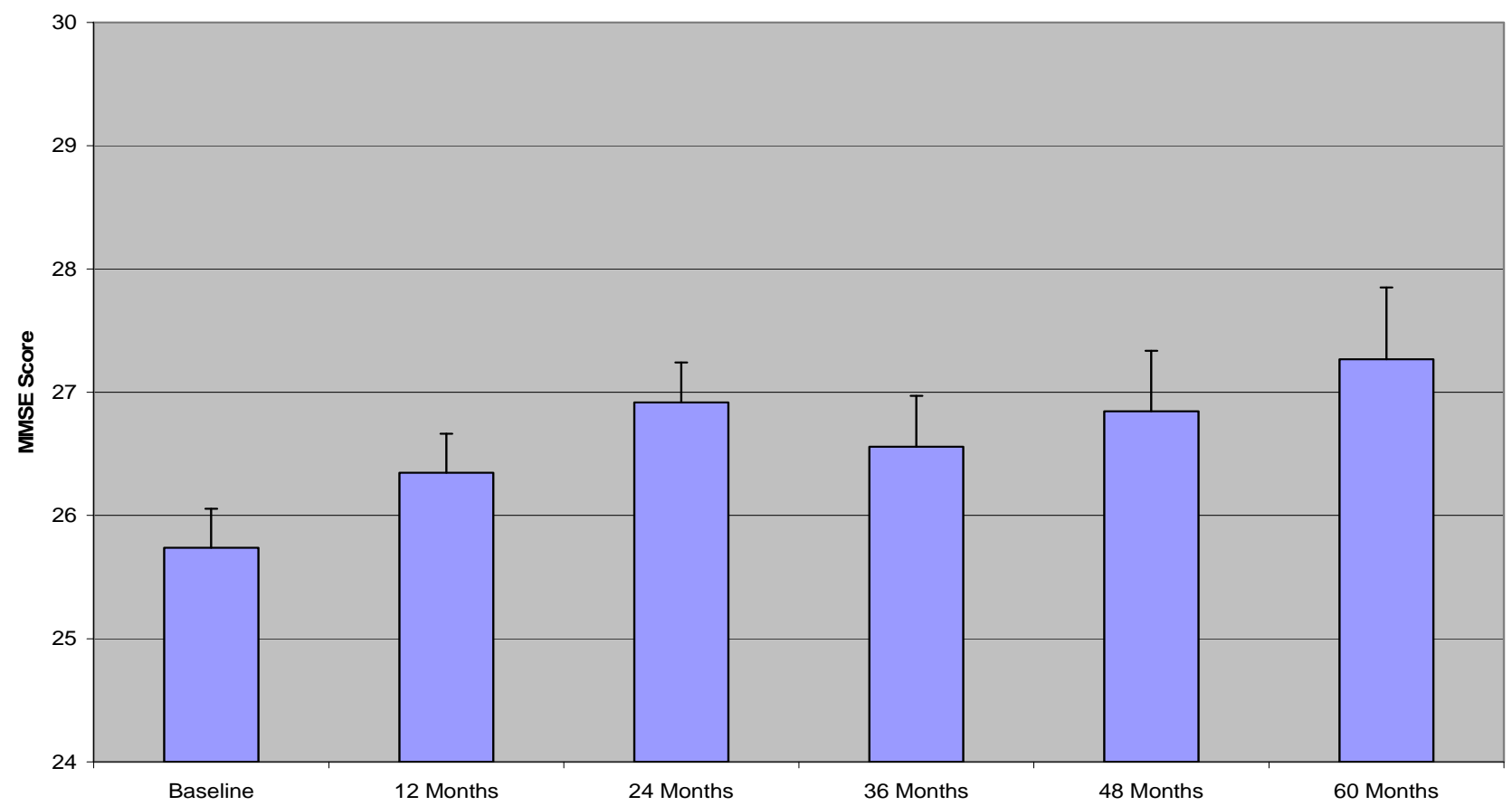

Figure 2. MMSE (Mini mental status exam). Data are presented as MEAN \pm SEM.

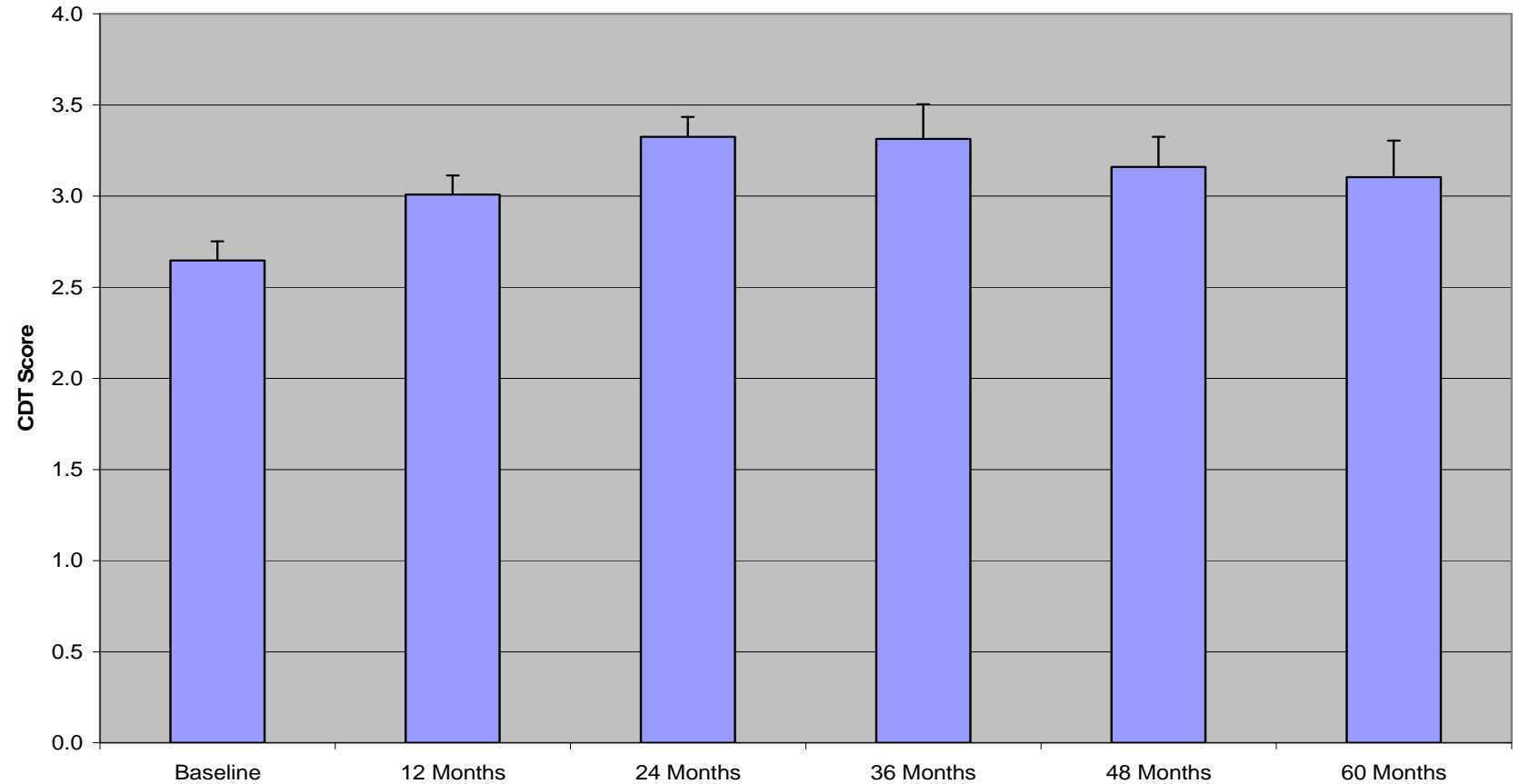

Figure 3. CDT (Clock drawing test). Data are presented as MEAN \pm SEM.

on CBF using ApoE4 transgenic mice compared to age-matched wild-type (WT) mice by use of [(14) C] iodoantipyrene autoradiography. ApoE4 associated factors reduced CBF gradually to create brain hypoperfusion relative to WT animals [54]. The differences in CBF are greatest in animals of age from 6-weeks to 12-months. Furthermore, transmission electron microscopy with colloidal gold immunocytochemistry showed that structural damage in young and aged endothelium microvessels of ApoE4 animals extended to the cytoplasm of perivascular cells, perivascular nerve terminals, hippocampal neurons, and glial cells. These abnormalities coexisted with mitochondrial structural alteration and mitochondrial DNA overproliferation and/or deletion in all 


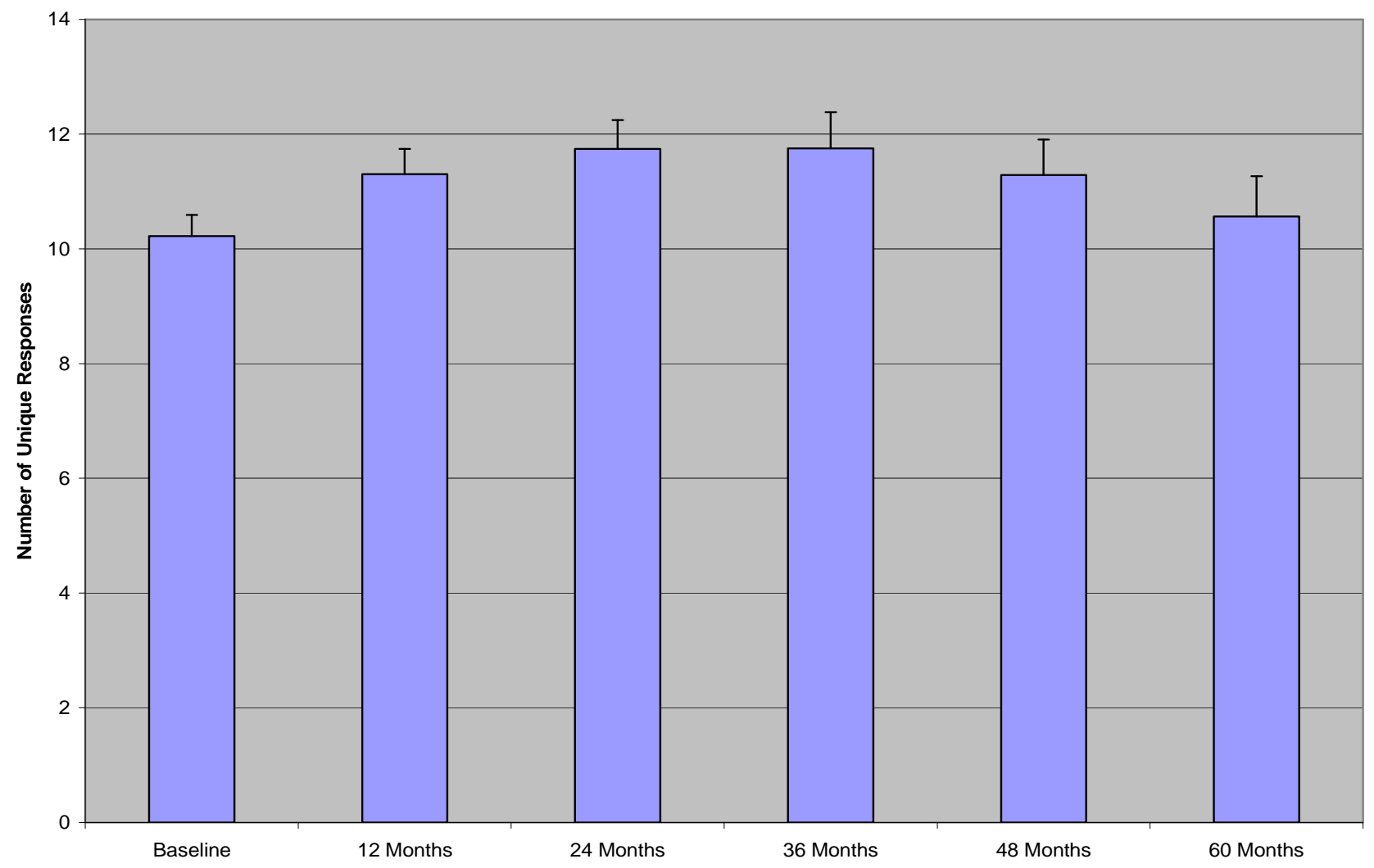

Figure 4. VFT (Animals). Data are presented as MEAN \pm SEM.

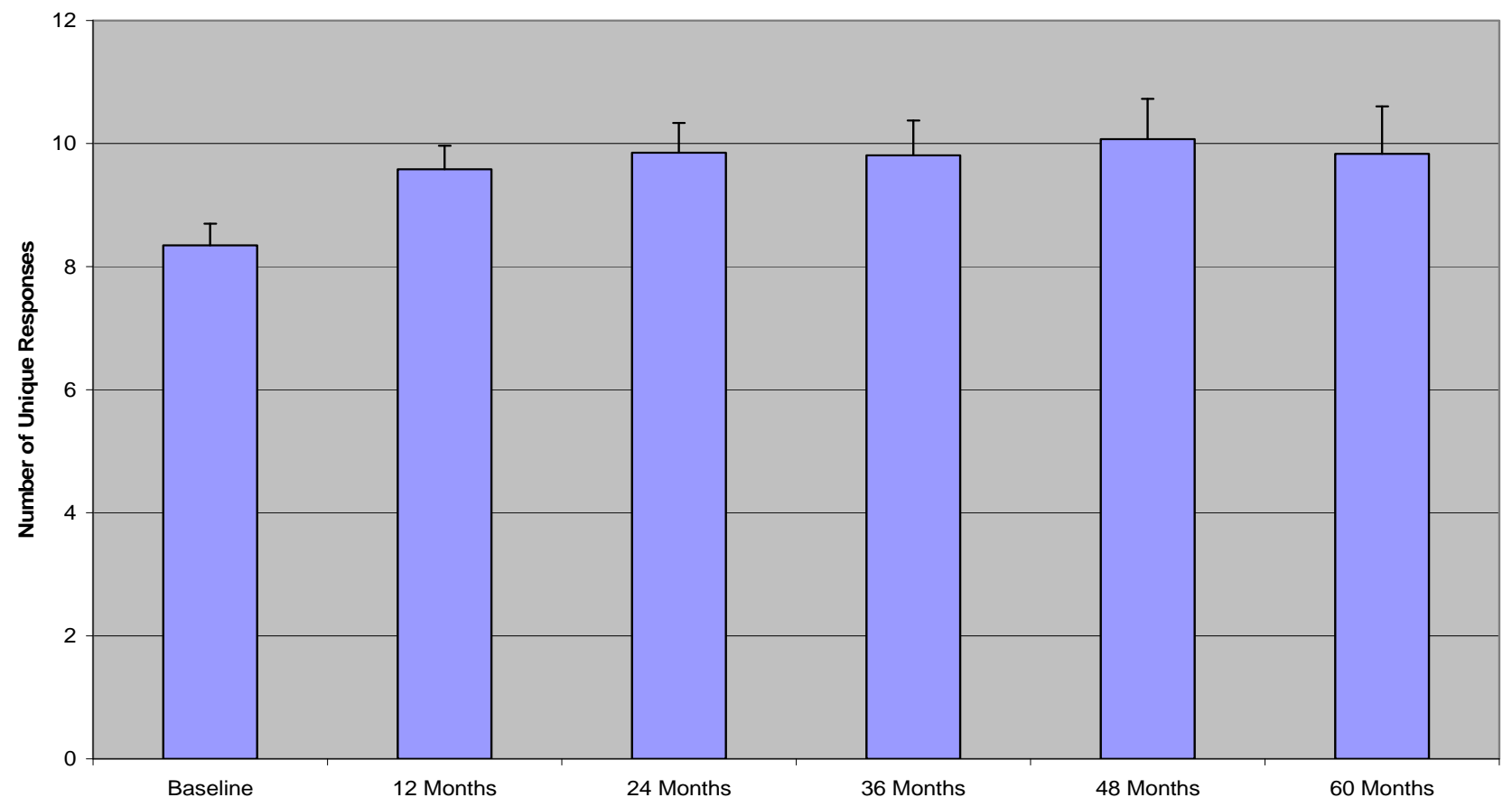

Figure 5. VFT (Letters). Data are presented as MEAN \pm SEM.

brain cellular compartments. Spatial and temporal memory tests showed a trend in improving cognitive function of ApoE4 mice fed selective mitochondrial antioxidants, acetyl-l-carnitine, and alpha-lipoic acid, which may have practical application in humans as well $[47,49,54]$.

Studies in rodents have shown that mental exercise 
enhances cerebrovasculature [55], induces neurogenesis [56] and synaptogenesis, increases hippocampal synaptic reactivity, and reduces brain $\mathrm{A} \beta$ deposition [57]. This basic research has served as the foundation for clinical implementation of an integrative treatment model: a combination of medication therapy and non-pharmacological modalities.

Clinical data related to the integrative treatment of $\mathrm{AD}$ is growing. A recent report by Chan et al. [32] showed an improvement in multiple domains of the neuropsychiatric inventory and maintenance of ADL in people in early stages of $\mathrm{AD}$, who were taking donepezil and a vitamin/ nutriceutical formulation for 12 months. Another report by Bottino et al. [30] indicated a positive treatment effects on the MMSE and the backward digit span as a result of a combined therapy (cognitive rehabilitation and rivastigmine) in $\mathrm{AD}$ patients for 5 months. A study by Rozzini et al. [31] demonstrated an improvement in memory, abstract reasoning, and depression in a longitudinal, 12 month, combined therapy (cognitive rehabilitation and cholinesterase inhibitors) study. Reguena et al. [58] used a combination therapy model (medication and cognitive training) and showed an improvement in cognitive functions by the end of 12 months of therapy. By the end of 24 months of the treatment, this effect diminished. Chapman et al. [59] studied the slowing rates of verbal and functional decline in $\mathrm{AD}$ patients, after 8 weeks of cognitive-communication stimulation and donepezil treatment. Erkroth-Bucher and Siberski [60] presented an improvement on the Dementia Rating Scale in mild AD patients after 6 weeks of cognitive training. Some of these patients were taking medications at the same time. There is no data to our knowledge related to the implementation of a multi-modal, integrative treatment in which many pharmacological and non-pharmacological modalities were used concurrently.

Our goal was to incorporate a set of non-pharmacological modalities to use simultaneously with standard pharmacological therapy. There was no consensus about non-pharmacological intervention modalities in literature. Based on emerging data related to hypoperfusion in the brain of demented individuals and its contribution to brain hypoxia, oxidative stress, and mitochondria failure, we developed the B-BAP ${ }^{\mathrm{TM}}$. At the heart of the program are hand and finger exercises, which increase regional CBF [61-65]. This fact has been well described in the literature but has not been seen in widespread clinical practice. Cognitive training has also been shown to increase CBF in healthy subjects [66]. To our knowledge, this program is the first attempt to use hand exercises for possible restoration of brain blood flow in chronic neurodegenerative disease, such as $\mathrm{AD}$.

The present material is a part of this ongoing, naturalistic treatment study. A comparison with similar studies could not be done due to the lack of literature of any study with a similar objective, except our previous 24and 36-month studies [35,36].

These studies have demonstrated the preservation and improvement of cognitive functions in people who have actively participated in the treatment. The maximum cognitive function improvements were seen after the first 6 months of treatment in the 24 month study (first cohort), and by 24 months of treatment in the 36 month study (second cohort). One possible explanation for the difference between the two cohorts is that patients from the first cohort had a younger average age and less cardiovascular pathology enabling them to respond to treatment earlier.

In the present study (third cohort), the maximum cognitive improvements were observed by 24 months on the treatment. The performance on all tasks did not decline but improved significantly (MMSE, Cognistat-Attention, Cognistat-Judgment, and RFFT-Total Unique Designs).

It was noted through performance, that brief cognitive tasks (MMSE, CDT, Cognistat, and VFT) demonstrated significant improvement for the first 36 months of the treatment. At the same time, the continuous performance tasks (Ruff 2 \& 7 SAT, WLMLT and RFFT) remained around the baseline. We suggest that brief cognitive tasks had a shorter administration time, and most likely activated a limited amount of networks that were less dependent on already compromised cerebral blood circulation. The continuous performance tasks had a longer administration time, and probably activated an extended numbers of networks which may have more heavily relied on an already limited supply of blood and oxygen going into the brain. This suggestion may warrant a separate investigation.

\subsection{Strengths of the Study}

There are several important strengths of this study. First, the primary strength of this ongoing, self-controlled, naturalistic study is that it is a real life outcome study whose longitudinal design closely reflects actual clinical practices. In our study, cognitive performance was tested every year during regular office visits for the entire period of 60 months.

Second, our study is unique in its simultaneous usage of the combination of pharmacological treatment with a set of comprehensive non-pharmacological interventions impacting brain function.

Third, the non-pharmacological part of the program was designed with fragile patients in mind, for long term, indefinite usage in the office and at home.

\subsection{Limitations of the Study}

Our study has certain limitations. First, depression was 
only clinically diagnosed, without using any psychological scales, such as Beck or Zung Depression and Anxiety scales or the Activities of Daily Living questionnaires. Secondly, there were some patients (approximately 30\%) who missed yearly retesting procedures and were tested in the following year. The third limitation is that there is no reliable information about the patients' compliance with medications, vitamins, physical exercises, and memory training. Fourth, data about concomitant medications were not always available. Fifth, the test results raters were non-blinded, and therefore there is the possibility that the raters were unintentionally biased to see positive results. Finally, a control group was not present as this would be practically impossible because of ethical, social, and personal issues related to the standards of the care for the treatment of the selected demented and depressed patients for this duration of time.

Despite these limitations, the data in this study demonstrates the effectiveness of a combined, multifaceted treatment approach and supports the notion that even today, there are many available venues in routine outpatient practice to prevent cognitive decline in medically ill, clinically depressed/demented patients over a long period of the time (60 months). The results of this observational study are encouraging. The cognitive function stabilization in this group was demonstrated consistently year by year, during the entire observation period. At this point, it is not possible to separate the role of each integrative treatment modality in preservation of cognitive decline in dementia/depression [67-69].

Based on this study, combination therapy can have an effect on disease progression and stabilization of cognitive function in mild to moderate dementia patients, even in severely medically ill patients with depression. One of the plausible explanations is a partial restoration of brain blood circulation, oxygen/nutrient flow, and the removal of oxidative stress products via the activation of a NO dependent pathway. Functional imaging studies, quantitative EEG, and NO assays would help to confirm these suggestions in the future.

\section{CONCLUSIONS}

The integrative treatment model described here has been effective in the preservation of cognitive functions in severely medically ill, demented, depressed seniors for period of 60 months.

As a new practical framework for treatment of patients with dementia and depression, it is important to include medical office visits, coupled with lifelong home based activities. Late age interventions appear to have positive effects in the treatment of the elderly. Going forward, prospective studies with combined therapy for "real life" patients with $\mathrm{AD} /$ dementia are necessary [67-69].

\section{ACKNOWLEDGEMENTS}

This study was supported by the Stress Relief and Memory Training Center, Brooklyn, New York, NY USA and “GALLY” International Biomedical Research Institute, Inc., San Antonio, TX, USA. We are very grateful for Ms. Galina Alieva for her editorial work throughout the preparation of this manuscript.

\section{REFERENCES}

[1] Aliev, G., Palacios, H.H., Lipsitt, A.E., Fischbach, K., Lamb, B.T., Obrenovich, M.E., Morales, L., Gasimov, E. and Bragin, V. (2009) Nitric oxide as an initiator of brain lesions during the development of Alzheimer disease. Neurotoxicity Research, 16, 293-305. doi:10.1007/s12640-009-9066-5

[2] Daulatzai, M.A. (2010) Early stages of pathogenesis in memory impairment during normal senescence and Alzheimer's disease. Journal of Alzheimer's Disease, 20, 355-367.

[3] De La Torre, J.C. (2008) Pathophysiology of neuronal energy crisis in Alzheimer's disease. Neurodegenerative Diseases, 5, 126-132. doi:10.1159/000113681

[4] Swerdlow, R.H. (2007) Pathogenesis of Alzheimer's disease. Journal of Clinical Interventions in Aging, 2, 347359.

[5] Weller, R.O., Subash, M., Preston, S.D., Mazanti, I. and Carare, R.O. (2008) Perivascular drainage of amyloidbeta peptides from the brain and its failure in cerebral amyloid angiopathy and Alzheimer's disease. Brain Pathology, 18, 253-266. doi:10.1111/j.1750-3639.2008.00133.x

[6] Querfurth, H.W. and LaFerla, F.M. (2010) Alzheimer's disease. The New England Journal of Medicine, 362, 329344. doi:10.1056/NEJMra0909142

[7] Swerdlow, R.H. and Khan, S.M. (2009) The Alzheimer's disease mitochondrial cascade hypothesis: An update. Experimental Neurology, 218, 308-315. doi:10.1016/j.expneurol.2009.01.011

[8] Helzner, E.P., Luchsinger, J.A., Scarmeas, N., Cosentino, S., Brickman, A.M., Glymour, M.M. and Stern, Y. (2009) Contribution of vascular risk factors to the progression in Alzheimer disease. Archives of Neurology, 66, 343-348. doi:10.1001/archneur.66.3.343

[9] Hanada, K., Hosono, M., Kudo, T., Hitomi, Y., Yagyu, Y., Kirime, E., Komeya, Y., Tsujii, N., Hitomi, K. and Nishimura, Y. (2006) Regional cerebral blood flow in the assessment of major depression and Alzheimer's disease in the early elderly. Nuclear Medicine Communications, 27, 535-541. doi:10.1097/00006231-200606000-00010

[10] Nobler, M.S., Pelton, G.H. and Sackeim, H.A. (1999) Cerebral blood flow and metabolism in late-life depression and dementia. Journal of Geriatric Psychiatry and Neurology, 12, 118-127. doi:10.1177/089198879901200305

[11] Henry-Feugeas, M.C. (2009) Assessing cerebrovascular contribution to late dementia of the Alzheimer's type: The role of combined hemodynamic and structural MR analysis. Journal of the Neurological Sciences, 283, 44- 


\section{8. doi:10.1016/j.jns.2009.02.325}

[12] Johnson, N.A., Jahng, G.H., Weiner, M.W., Miller, B.L., Chui, H.C., Jagust, W.J., Gorno-Tempini, M.L. and Schuff, N. (2005) Pattern of cerebral hypoperfusion in Alzheimer disease and mild cognitive impairment measured with arterial spin-labeling MR imaging: Initial experience. $R a$ diology, 234, 851-859. doi:10.1148/radiol.2343040197

[13] Kataoka, K., Hashimoto, H., Kawabe, J., Higashiyama, S., Akiyama, H., Shimada, A., Kai, T., Inoue, K., Shiomi, S. and Kiriike, N. (2010) Frontal hypoperfusion in depressed patients with dementia of Alzheimer type demonstrated on 3DSRT. Psychiatry and Clinical Neurosciences, 64, 293-298. doi:10.1111/j.1440-1819.2010.02083.x

[14] Prins, N. D., Van Straaten, E.C., van Dijk, E.J., Simoni, M., van Schijndel, R.A., Vrooman, H.A., Koudstaal, P.J., Scheltens, P., Breteler, M.M. and Barkhof, F. (2004) Measuring progression of cerebral white matter lesions on MRI: Visual rating and volumetrics. Neurology, 62, 1533-1539.

[15] Peers, C., Dallas, M.L., Boycott, H.E., Scragg, J.L., Pearson, H.A. and Boyle, J.P. (2009) Hypoxia and neurodegeneration. Annals of the New York Academy of Sciences, 1177, 169-177. doi:10.1111/j.1749-6632.2009.05026.x

[16] Peers, C., Pearson, H.A. and Boyle, J.P. (2007) Hypoxia and Alzheimer's disease. Essays in Biochemistry, 43, 153164. doi:10.1042/BSE0430153

[17] Zhang, X. and Le, W. (2010) Pathological role of hypoxia in Alzheimer's disease. Experimental Neurology, 223, 299-303. doi:10.1016/j.expneurol.2009.07.033

[18] Prins, N.D., van Dijk, E.J., den Heijer, T., Vermeer, S.E., Koudstaal, P.J., Oudkerk, M., Hofman, A. and Breteler, M.M. (2004) Cerebral white matter lesions and the risk of dementia. Archives of Neurology, 61, 1531-1534. doi:10.1001/archneur.61.10.1531

[19] Bennett, S., Grant, M.M. and Aldred, S. (2009) Oxidative stress in vascular dementia and Alzheimer's disease: A common pathology. Journal of Alzheimer's Disease, 17, 245-257.

[20] Aliev, G., Palacios, H.H., Walrafen, B., Lipsitt, A.E., Obrenovich, M.E. and Morales, L. (2009) Brain mitochondria as a primary target in the development of treatment strategies for Alzheimer disease. The International Journal of Biochemistry \& Cell Biology, 41, 1989-2004. doi:10.1016/j.biocel.2009.03.015

[21] Andrade, C. and Radhakrishnan, R. (2009) The prevention and treatment of cognitive decline and dementia: An overview of recent research on experimental treatments. Indian Journal of Psychiatry, 51, 12-25.

[22] Acevedo, A. and Loewenstein, D.A. (2007) Nonpharmacological cognitive interventions in aging and dementia. Journal of Geriatric Psychiatry and Neurology, 20, 239249. doi:10.1177/0891988707308808

[23] Adamsa, J., Luia, C. and McLaughlina, D. (2009) The use of complementary and alternative medicine in later life. Reviews in Clinical Gerontology, Cambridge University Press, Cambridge, 227-236.

[24] Goh, J.O. and Park, D.C. (2009) Neuroplasticity and cog- nitive aging: The scaffolding theory of aging and cognition. Restorative Neurology and Neuroscience, 27, 391403.

[25] Kuo, M.F., Grosch, J., Fregni, F., Paulus, W. and Nitsche, M.A. (2007) Focusing effect of acetylcholine on neuroplasticity in the human motor cortex. The Journal of Neuroscience, 27, 14442-14447.

doi:10.1523/JNEUROSCI.4104-07.2007

[26] Middleton, L.E. and Yaffe, K. (2010) Targets for the prevention of dementia. Journal of Alzheimer's Disease, 20, 915-924.

[27] Zec, R.F. and Burkett, N.R. (2008) Non-pharmacological and pharmacological treatment of the cognitive and behavioral symptoms of Alzheimer disease. NeuroRehabilitation, 23, 425-438.

[28] Burns, A., Gauthier, S. and Perdomo, C. (2007) Efficacy and safety of donepezil over 3 years: An open-label, multicentre study in patients with Alzheimer's disease. International Journal of Geriatric Psychiatry, 22, 806-812. doi:10.1002/gps.1746

[29] Persson, C.M., Wallin, A.K., Levander, S. and Minthon, L. (2009) Changes in cognitive domains during three years in patients with Alzheimer's disease treated with donepezil. BMC Neurology, 9, 7. doi:10.1186/1471-2377-9-7

[30] Bottino, C.M., Carvalho, I.A., Alvarez, A.M., Avila, R., Zukauskas, P.R., Bustamante, S.E., Andrade, F.C., Hototian, S.R., Saffi, F. and Camargo, C.H. (2005) Cognitive rehabilitation combined with drug treatment in Alzheimer's disease patients: A pilot study. Clinical Rehabilitation, 19, 861-869. doi:10.1191/0269215505cr911oa

[31] Rozzini, L., Costardi, D., Chilovi, B.V., Franzoni, S., Trabucchi, M. and Padovani, A. (2007) Efficacy of cognitive rehabilitation in patients with mild cognitive impairment treated with cholinesterase inhibitors. International Journal of Geriatric Psychiatry, 22, 356-360. doi:10.1002/gps.1681

[32] Chan, A., Paskavitz, J., Remington, R., Rasmussen, S. and Shea, T.B. (2008) Efficacy of a vitamin/nutriceutical formulation for early-stage Alzheimer's disease: A 1-year, open-label pilot study with an 16-month caregiver extension. American Journal of Alzheimer's Disease and Other Dementias, 23, 571-585. doi:10.1177/1533317508325093

[33] Heyn, P. (2003) The effect of a multisensory exercise program on engagement, behavior, and selected physiological indexes in persons with dementia. American Journal of Alzheimer's Disease and Other Dementias, 18, 247-251. doi:10.1177/153331750301800409

[34] Yu, F., Kolanowski, A.M., Strumpf, N.E. and Eslinger, P.J. (2006) Improving cognition and function through exercise intervention in Alzheimer's disease. Journal of Nursing Scholarship, 38, 358-365. doi:10.1111/j.1547-5069.2006.00127.x

[35] Bragin, V., Chemodanova, M., Dzhafarova, N., Bragin, I., Czerniawski, J.L. and Aliev, G. (2005) Integrated treatment approach improves cognitive function in demented and clinically depressed patients. American Journal of Alzheimer's Disease and Other Dementias, 20, 21-26. doi:10.1177/153331750502000103 
[36] Bragin, V., Chemodanova, M., Dzhafarova, N., Bragin, I., Chernyavskyy, P. and Aliev, G. (2009) Preservation of cognitive functioning in depressed, demented geriatric patients with cardiovascular risk factors: An ongoing 3year naturalistic study. Alzheimer's \& Dementia, 5, 320. doi:10.1016/j.jalz.2009.04.517

[37] Bragin, V. (2007) How to activate your brain. Authorhouse, Bloominton.

[38] Bragin, V., Chemodanova, M., Vaysman, V., Bragin, I., Chernyavskyy, P., Grinayt, E. and Aliev, G. (2009) Preservation of learning abilities in people with dementia and depression with different level of cognitive impairment. Alzheimer's \& Dementia, 5, 322. doi:10.1016/j.jalz.2009.04.523

[39] Bragin, V., Chemodanova, M., Vaysman, V., Bragin, I., Grinayt, E. and Ruditser, M. (2008) N-back task to tailor memory training protocols for patients with depression and dementia. Alzheimer's Association International Conference on Alzheimer's Disease, Chicago, 26-31 July 2008, 496-497.

[40] Folstein, M.F., Folstein, S.E. and McHugh, P.R. (1975) "Mini-mental state." A practical method for grading the cognitive state of patients for the clinician. Journal of Psychiatric Research, 12, 189-198. doi:10.1016/0022-3956(75)90026-6

[41] Kiernan, R., Mueller, J. and Langston, W. (2002) Cognistat (neurobehavioral cognitive status examination). Psychological Assessment Resources, Odessa, FLA.

[42] Ruff, R. and Allen, C. (2002) Ruff 2 \& 7 selective attention test. Psychological Assessment Resources, Odessa.

[43] Ruff, R. (2002) Ruff Figural Fluency Test (RFFT). Psychological Assessment Resources, Odessa.

[44] SPSS Base 17 Applications Guide (2009) SPSS Inc., Chicago.

[45] Hall, C.B., Lipton, R.B., Sliwinski, M., Katz, M.J., Derby, C.A. and Verghese, J. (2009) Cognitive activities delay onset of memory decline in persons who develop dementia. Neurology, 73, 356-361. doi:10.1212/WNL.0b013e3181b04ae3

[46] Middleton, L.E. and Yaffe, K. (2009) Promising strategies for the prevention of dementia. Archives of Neurology, 66, 1210-1215. doi:10.1001/archneurol.2009.201

[47] Aliev G., Li, Y., Palacios, H.H. and Obrenovich, M.E. (2011) Oxidative stress induced mitochondrial DNA deletion as a hallmark for the drug development in the context of the cerebrovascular diseases. Recent Patents on Cardiovascular Drug Discovery, 6, 222-241.

[48] Kobayashi, S., Tateno, M., Utsumi, K., Takahashi, A., Saitoh, M., Morii, H., Fujii, K. and Teraoka, M. (2008) Quantitative analysis of brain perfusion SPECT in Alzheimer's disease using a fully automated regional cerebral blood flow quantification software, 3DSRT. Journal of the Neurological Sciences, 264, 27-33. doi:10.1016/j.jns.2007.07.015

[49] Aliev, G., Liu, J., Shenk, J.C., Fischbach, K., Pacheco, G.J., Chen, S.G., Obrenovich, M.E., Ward, W.F., Richardson, A.G., Smith, M. A., Gasimov, E., Perry, G. and Ames, B.N. (2009) Neuronal mitochondrial amelioration by feeding acetyl-L-carnitine and lipoic acid to aged rats. Journal of Cellular and Molecular Medicine, 13, 320-333. doi:10.1111/j.1582-4934.2008.00324.x

[50] Milgram, N.W., Araujo, J.A., Hagen, T.M., Treadwell, B.V. and Ames, B.N. (2007) Acetyl-L-carnitine and alpha-lipoic acid supplementation of aged beagle dogs improves learning in two landmark discrimination tests. The FASEB Journal, 21, 3756-3762. doi:10.1096/fj.07-8531com

[51] Head, E., Nukala, V.N., Fenoglio, K.A., Muggenburg, B.A., Cotman, C.W. and Sullivan, P.G. (2009) Effects of age, dietary, and behavioral enrichment on brain mitochondria in a canine model of human aging. Experimental Neurology, 220, 171-176. doi:10.1016/j.expneurol.2009.08.014

[52] Pop, V., Head, E., Hill, M.A., Gillen, D., Berchtold, N.C., Muggenburg, B.A., Milgram, N.W., Murphy, M.P. and Cotman, C.W. (2010) Synergistic effects of long-term antioxidant diet and behavioral enrichment on beta-amyloid load and non-amyloidogenic processing in aged canines. The Journal of Neuroscience, 30, 9831-9839. doi:10.1523/JNEUROSCI.6194-09.2010

[53] Manukhina, E.B., Goryacheva, A.V., Barskov, I.V., Viktorov, I.V., Guseva, A.A., Pshennikova, M.G., Khomenko, I.P., Mashina, S.Y., Pokidyshev, D.A. and Malyshev, I.Y. (2010) Prevention of neurodegenerative damage to the brain in rats in experimental Alzheimer's disease by adaptation to hypoxia. Neuroscience and Behavioral Physiology, 40, 737-743. doi:10.1007/s11055-010-9320-6

[54] Shenk, J.C., Liu, J., Fischbach, K., Xu, K., Puchowicz, M., Obrenovich, M.E., Gasimov, E., Alvarez, L.M., Ames, B.N., Lamanna, J.C. and Aliev, G. (2009) The effect of acetyl-L-carnitine and R-alpha-lipoic acid treatment in ApoE4 mouse as a model of human Alzheimer's disease. Journal of the Neurological Sciences, 283, 199-206. doi:10.1016/j.jns.2009.03.002

[55] Black, J.E., Sirevaag, A.M. and Greenough, W.T. (1987) Complex experience promotes capillary formation in young rat visual cortex. Neuroscience Letters, 83, 351-355. doi:10.1016/0304-3940(87)90113-3

[56] Brown, J., Cooper-Kuhn, C.M., Kempermann, G., Van Praag, H., Winkler, J., Gage, F.H. and Kuhn, H.G. (2003) Enriched environment and physical activity stimulate hippocampal but not olfactory bulb neurogenesis. European Journal of Neuroscience, 17, 2042-2046. doi:10.1046/j.1460-9568.2003.02647.x

[57] Cracchiolo, J.R., Mori, T., Nazian, S.J., Tan, J., Potter, H. and Arendash, G.W. (2007) Enhanced cognitive activeity-Over and above social or physical activity-Is required to protect Alzheimer's mice against cognitive impairment, reduce Abeta deposition, and increase synaptic immunoreactivity. Neurobiology of Learning and Memory, 88, 277-294. doi:10.1016/j.nlm.2007.07.007

[58] Requena, C., Maestu, F., Campo, P., Fernandez, A. and Ortiz, T. (2006) Effects of cholinergic drugs and cognitive training on dementia: 2-year follow-up. Dementia and Geriatric Cognitive Disorders, 22, 339-345. doi:10.1159/000095600 
[59] Chapman, S.B., Weiner, M.F., Rackley, A., Hynan, L.S. and Zientz, J. (2004) Effects of cognitive-communication stimulation for Alzheimer's disease patients treated with donepezil. Journal of Speech, Language, and Hearing Research, 47, 1149-1163. doi:10.1044/1092-4388(2004/085)

[60] Eckroth-Bucher, M. and Siberski, J. (2009) Preserving cognition through an integrated cognitive stimulation and training program. American Journal of Alzheimer's Disease and Other Dementias, 24, 234-245. doi:10.1177/1533317509332624

[61] Kawashima, R., Itoh, H., Ono, S., Satoh, K., Furumoto, S., Gotoh, R., Koyama, M., Yoshioka, S., Takahashi, T., Takahashi, K., Yanagisawa, T. and Fukuda, H. (1996) Changes in regional cerebral blood flow during selfpaced arm and finger movements. A PET study. Brain Research, 716, 141-148. doi:10.1016/0006-8993(96)00032-7

[62] Kawashima, R., Matsumura, M., Sadato, N., Naito, E., Waki, A., Nakamura, S., Matsunami, K., Fukuda, H. and Yonekura, Y. (1998) Regional cerebral blood flow changes in human brain related to ipsilateral and contralateral complex hand movements-A PET study. European Journal of Neuroscience, 10, 2254-2260. doi:10.1046/j.1460-9568.1998.00237.x

[63] Khalsa, D.S., Amen, D., Hanks, C., Money, N. and Newberg, A. (2009) Cerebral blood flow changes during chanting meditation. Nuclear Medicine Communications, 30, 956-961. doi:10.1097/MNM.0b013e32832fa26c

[64] Roland, P.E., Meyer, E., Shibasaki, T., Yamamoto, Y.L. and Thompson, C.J. (1982) Regional cerebral blood flow changes in cortex and basal ganglia during voluntary movements in normal human volunteers. Journal of $\mathrm{Neu}$ - rophysiology, 48, 467-480.

[65] Van Mier, H., Tempel, L.W., Perlmutter, J.S., Raichle, M.E. and Petersen, S.E. (1998) Changes in brain activity during motor learning measured with PET: Effects of hand of performance and practice. Journal of Neurophysiology, 80, 2177-2199.

[66] Mozolic, J.L., Hayasaka, S. and Laurienti, P.J. (2010) A cognitive training intervention increases resting cerebral blood flow in healthy older adults. Frontiers in Human Neuroscience, 4, 16. doi:10.3389/neuro.09.016.2010

[67] Bragin, V. and Aliev, G. (2010) Progression of cognitive function after integrated treatment approach in demented and clinically depressed patients. In: Aliev, G., et al., Eds., The Role of Oxidative Stress, Mitochondria Failure and Cellular Hypoperfusion in the Pathobiology of Alzheimer Disease, Research Signpost, Inc., Trivandrum, 317-325.

[68] Palacios, H.H., Yendluri, B.B., Parvathaneni, K., Shadlinski, V.B., Obrenovich, M.E., Leszek, J., Gokhman, D., Gasiorowski, K., Bragin, V. and Aliev, G. (2011) Mitochondrion-specific antioxidants as drug treatments for Alzheimer disease. CNS \& Neurological DisordersDrug Targets, 10, 149-162.

[69] Aliev, G. (2011) Oxidative stress induced-metabolic imbalance, mitochondrial failure, and cellular hypoperfusion as primary pathogenetic factors for the development of Alzheimer disease which can be used as a alternate and successful drug treatment strategy: Past, present and future. CNS \& Neurological Disorders-Drug Targets, 10, 147-148. 